\title{
THE ROLE OF THE CYCLIC DEPSIPEPTIDE RINGS IN ANTIBIOTICS
}

\author{
Fusao Takusagawa
}

Department of Chemistry, University of Kansas

Lawrence, Kansas 66045, U.S.A.

(Received for publication June 24, 1985)

\begin{abstract}
The physical and biological roles of the cyclic depsipeptides of actinomycin, quinomycin and triostin antibiotic families are proposed by examining the crystal structures of $\mathrm{d}(\mathrm{GC})-$ actinomycin D and d(CGTACG)-triostin A. The analyses suggest that not only are DNAamino acid hydrogen-bonding and chromophore-base pair stacking crucially important for DNA-antibiotic interaction, but also that the unique structure of the cyclic depsipeptides (the perfect hydrophobic character of the inner surface) is equally necessary to insure that these interactions are directed, unambiguous and screened from interference by solvent. Beyond this, the characteristic nature of the outer surfaces suggests a further hypothesis for the biological role of the cyclic depsipeptide rings; when the antibiotics bind in the region around the pause or rho-dependent termination sites on the DNA, the drugs actually terminate transcription by RNA polymerase and cause release of a premature RNA transcript. Termination is likely because the antibiotics carry five to six consecutive apparent $\mathrm{A} / \mathrm{T}$ sequences on the surface of the cyclic depsipeptide rings, thus presenting a deceptive termination signal to the polymerase.
\end{abstract}

Many naturally occurring antibiotics contain cyclic depsipeptides closed by an ester linkage. Some of these act by binding to DNA. The planar residue (chromophore) intercalates between the base pairs of DNA, and the other moiety binds to the surface of double helical DNA. The role of the planar residue is relatively well established, and it is also known that some amino acid residues in the depsipeptide ring are essential to bind the antibiotic to DNA by forming hydrogen bonds. However, although it is well recognized that the other amino acid residues are equally necessary to maintain the biological activity, the exact role of these residues is not well established. Thus, for example, it is extremely difficult to predict a priori precisely which chemical modification would be appropriate in order to increase the biological activity of the antibiotics isolated from nature.

We have proposed an important physical role of cyclic depsipeptide rings in DNA-drug interaction $^{1)}$ based on the crystal structure of $d(G C)$-actinomycin $D^{2)}$. In recent crystal structure determination of the d(CGTACG)-triostin $\mathrm{A}^{3)}$, the cyclic depsipeptide ring of the antibiotic displays a characteristic geometry extremely similar to that of actinomycin D. Now we confirm our previous proposal by use of this structure, and we further extend our previous proposal to include biological role for the cyclic depsipeptide rings. Our hypothesis explains the biological activity of these antibiotics, especially their extremely powerful, selective inhibition of DNA-directed RNA synthesis.

\section{Results and Discussion}

Since biological reactions take place in three-dimensional space, one of the best approaches for elucidating the role of the cyclic depsipeptides is to examine the three-dimensional structures of complexes between the antibiotic and DNA by X-ray crystal structure determinations. To date only two structures of this category have been reported; $d(\mathrm{GC})$-actinomycin $\mathrm{D}^{2)}$ and $\mathrm{d}(\mathrm{CGTACG})$-triostin $\mathrm{A}$ 
Fig. 1. The molecular formulas of actinomycin D and triostin A.

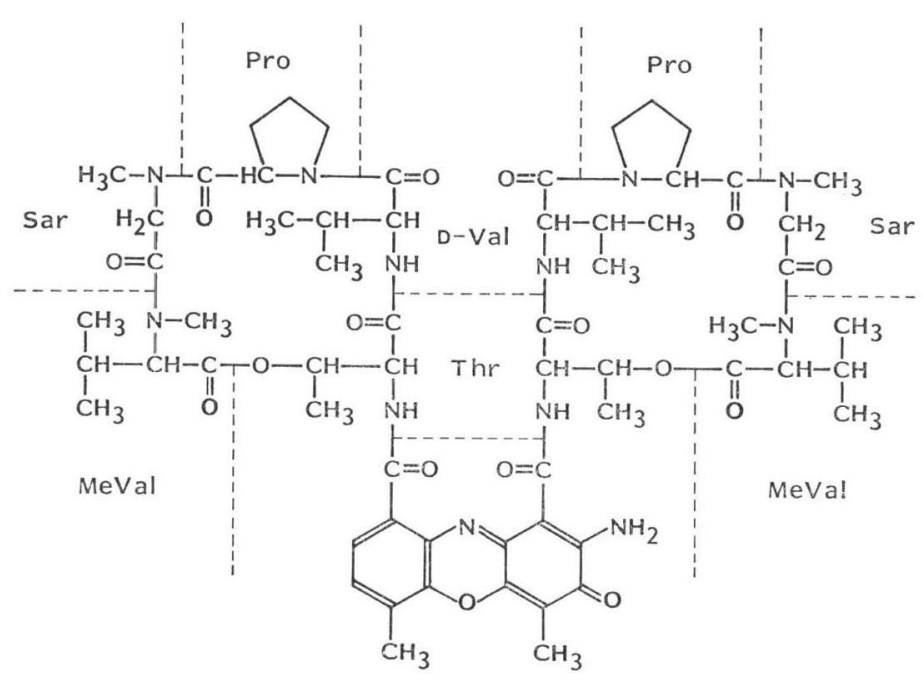

Actinomycin D

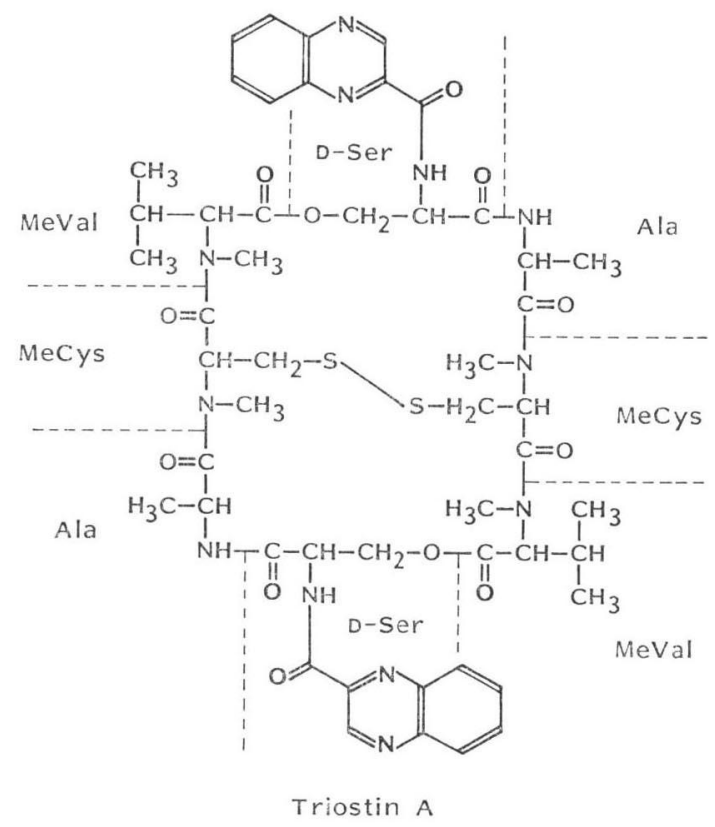

complexes $^{3)}$. Although the structure found in the crystal of $\mathrm{d}(\mathrm{GC})$-actinomycin D does not exactly show intercalation, the geometry of the binding site of this antibiotic can be considered as a general feature of intercalation of this antibiotic family. The model structure proposed by SoBELL and JAIN ${ }^{4)}$ based on the crystal structure of the deoxyguanosine-actinomycin D complex ${ }^{5)}$ indicates a quite similar geometry at the binding site. In the crystal structure of the d(CGTACG)-triostin A complex, the bisintercalator, triostin A, binds at the edge of the DNA fragment, and possibly for this reason, the idealized two-fold symmetry of the molecule is slightly distorted. One of the four hydrogen bonds between the antibiotic and DNA is missing, and one of the six carbonyl oxygen atoms is oriented in a 
Fig. 2. Schematic view of molecular structure of actinomycin D found in the crystal structure of its complex with $\mathrm{d}(\mathrm{GC})$.

The phenoxazone ring and base-pairs of DNA are shown by solid and open rectangles, respectively. The back-bones of cyclic depsipeptides and nucleotides are illustrated by solid lines, while the hydrogen bonds are indicated by dashed lines.

The carbonyl oxygen atoms of all residues, amide nitrogen atoms of threonine residues and characteristic methyl groups of $\mathrm{N}$-methyl-L-valine are marked by $\mathrm{O}, \mathrm{N}$ and $\mathrm{C}$, respectively. The $\mathrm{C}$ and $\mathrm{G}$ in base-pair boxes represent the cytosine and guanine bases, respectively. $\mathrm{N} 2$ and $\mathrm{N} 3$ belong to the guanine rings.
(a) View down on the cyclic depsipeptide rings.
(b) Side view of (a).

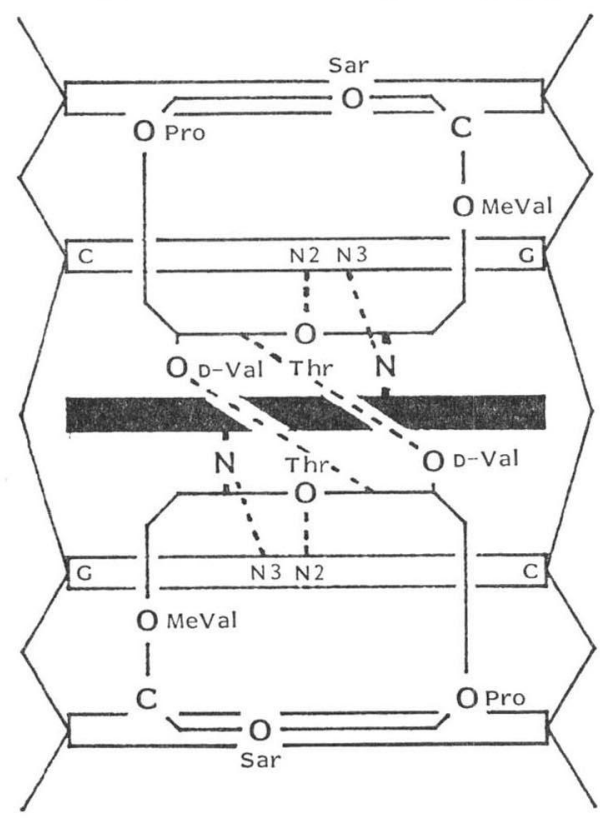

(a)

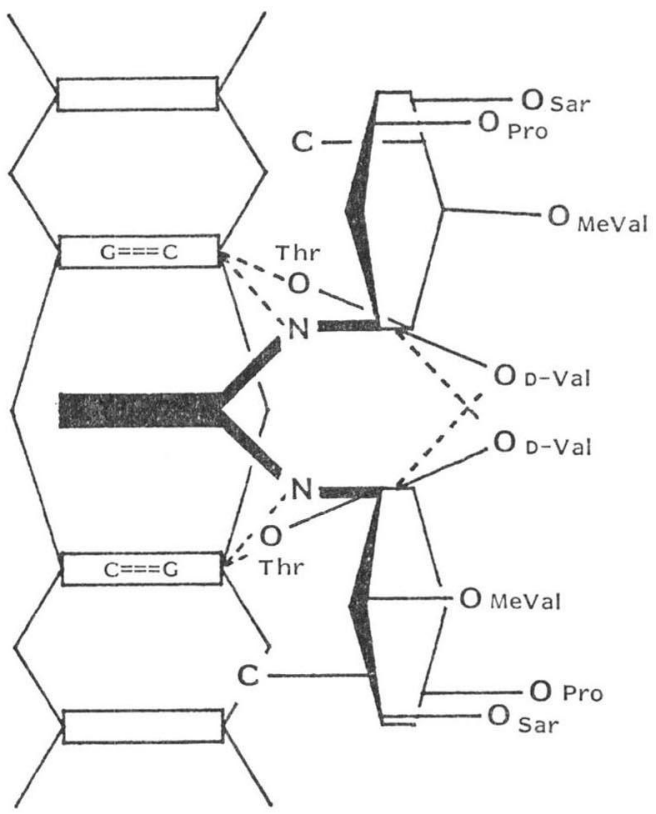

(b)

sense opposite to that expected. But the overall geometry of the binding site can be considered as a general feature of interaction between quinoxaline antibiotics and DNA. NMR studies support this geometry ${ }^{6)}$.

Physical Role of the Cyclic Depsipeptide Rings

The amino acid residues of depsipeptides found in actinomycin and triostin have two unique characteristics; some D-amino acid residues are present, and several of the amide nitrogens are blocked by hydrophobic groups (Fig. 1). This unusual pattern can be rationalized by careful analysis of the crystal structures of these antibiotic-oligonucleotide complexes.

As shown in Figs. 2 and 3, the amide hydrogen and carbonyl oxygen of the amino acid residue located at the middle of the molecules (L-threonine for actinomycin and L-alanine for triostin) form hydrogen bonds with guanine N3 and N2, respectively. The hydrophilic carbonyl oxygen atoms of the other residues are on the outer surface of the cyclic depsipeptide rings, away from the nucleic acid. The most hydrophobic groups of the rings are arranged so as to cover the DNA surface, the inner surfaces of the depsipeptide rings thus being perfectly hydrophobic in character and serving as an "umbrella" that effectively protects the essential hydrogen bonds between guanine N3 and N2 and the 
Fig. 3. Schematic view of molecular structure of triostin A found in the crystal structure of its complex with d(CGTACG).

The symbols are the same as in Fig. 2.

(a) View down on the cyclic depsipeptide ring. (b) Side view of (a).

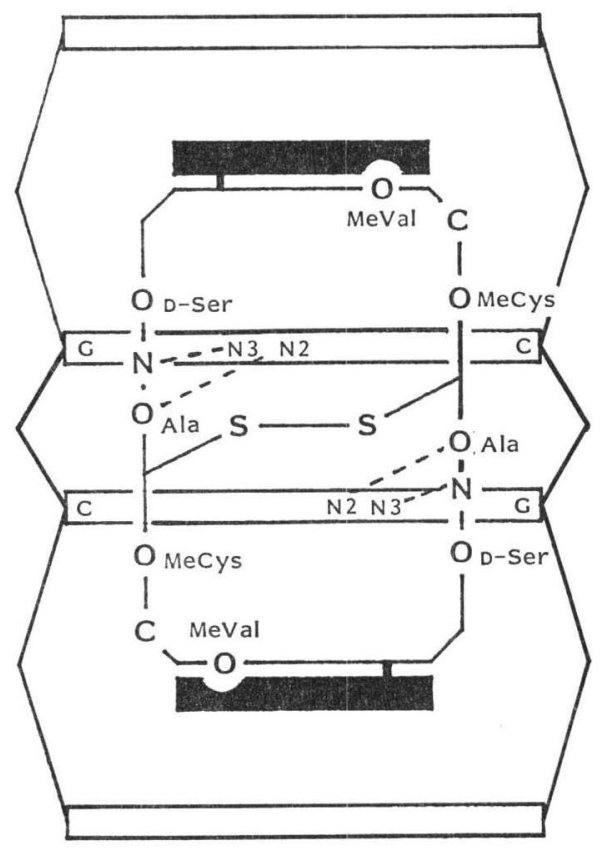

(a)

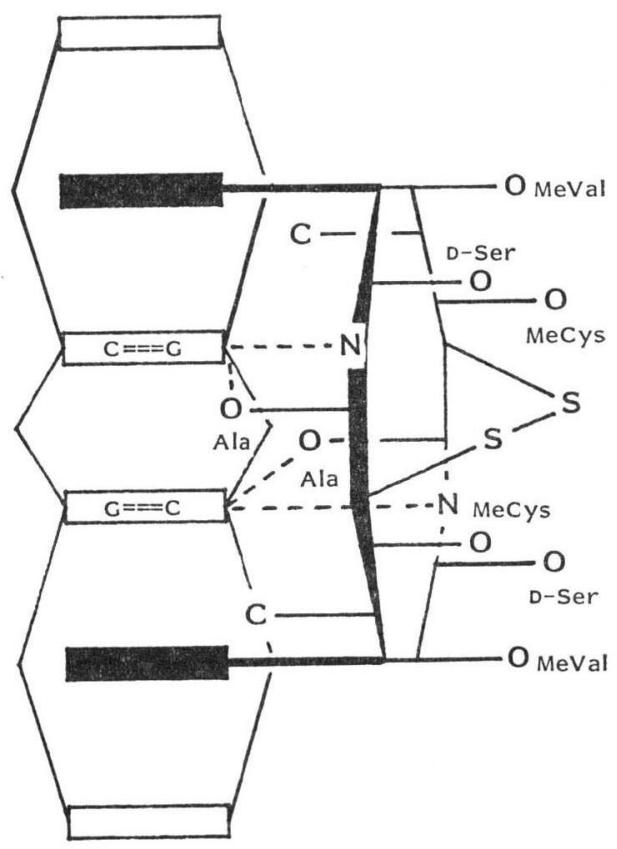

(b)

middle amino acid residue from a competitive interaction with water molecules. The methyl groups attached to the amide nitrogens of $\mathrm{N}$-methyl-L-valine residues in both antibiotics are located in exactly the right place to serve as a most effective "umbrella". Other features reduce substantially the mobility of the cyclic depsipeptide rings, promoting both the "umbrella effect" and the hydrogenbonding interaction with the DNA. These features are intramolecular hydrogen bonds between the cyclic depsipeptide rings in the case of actinomycin (Fig. 2) and a disulfide bridge in the case of triostin (Fig. 3).

As described above and illustrated in Figs. 2 and 3, the overall geometries of the cyclic depsipeptide rings of actinomycin and triostin are surprisingly similar in terms of the separation of hydrophilic groups (carbonyl oxygens) and hydrophobic groups (methyl and dimethyl groups). It can be seen that the configuration and conformation of each residue of the depsipeptide rings are critical to maintaining the specific drug-DNA interaction. For example, the overall effect of eliminating any or all of the hydrophobic groups from the amide nitrogens would be either (a) to allow solvent-DNA or solvent-drug hydrogen bonds to from preferentially to the DNA-drug ones; or (b) to cause some ambiguity in the DNA-drug binding since there would then be more drug hydrogen-bonding donors available for binding to the DNA. Indeed, a synthetic analogue, de- $N$-tetramethyltriostin $\mathrm{A}$, shows a different binding preference from triostin $\mathrm{A}$ itself ${ }^{7)}$. If the cross-bridge of triostin $\mathrm{A}$ is lacking, the molecule either fails to bind to DNA at all or binds with grossly altered characteristics. If one or both of the D-amino acids is epimerized to the L-form, the molecule is inactivated ${ }^{7,8)}$. Similarly, many attempts at chemical modification of the cyclic depsipeptide rings of actinomycins have resulted in 
Fig. 4. Hydrogen bonding pattern in A-T and G-C base-pairs.

The major and minor groove sides are at the top and bottom of each figure, respectively.

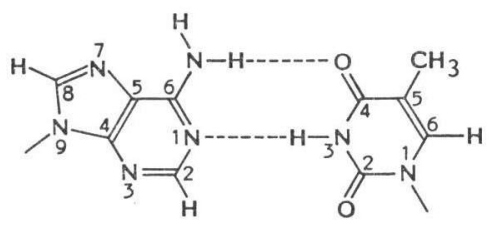

Adenine

Thymine

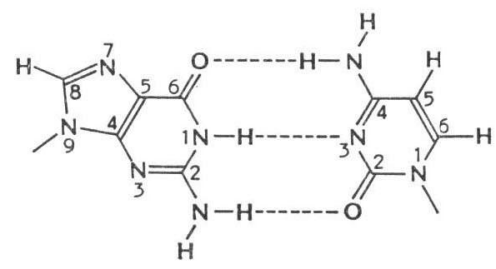

Guanine

Cytosine

loss of the antibiotic activity ${ }^{9)}$. In summary, this analysis suggests that not only are the DNA-amino acid hydrogen bonding and chromophore-base pair stacking crucially important for DNA-antibiotic interaction, but also that the unique structure of depsipeptide rings (the perfect hydrophobic character of the inner surfaces) is equally necessary to insure that these interactions are directed, unambiguous and screened from interference by solvent.

\section{Biological Role of the Cyclic Depsipeptide Ring}

As discussed above, the perfect hydrophobic characters of inner surfaces of cyclic depsipeptide rings are very important to bind the antibiotics on the DNA. How about the outer surfaces? Here we examine and discuss this point.

In the crystal structures of $\mathrm{d}(\mathrm{GC})$-actinomycin $\mathrm{D}$ and $\mathrm{d}$ (TACGTA)-triostin A complexes, the chromophore of actinomycin is intercalated into the GC sequence site, while the CG sequence is sandwiched between the two chromophores of triostin. These binding geometries suggest that the antibiotics bind at different sites of DNA. By the footprinting method, the actinomycin D and quinomycin A (identical structure with triostin A except for the cross-bridge mode: $-\mathrm{CH}\left(\mathrm{SCH}_{3}\right)-\mathrm{S}-\mathrm{CH}_{2}-$ ) are found to bind preferentially at $\mathrm{XGCY}(X \neq \mathrm{G} \text { and } \mathrm{Y} \neq \mathrm{C})^{10)}$ and $\mathrm{XCGT}(\mathrm{X}=\mathrm{A} \text { or } \mathrm{T})^{11)}$, respectively. It is obvious that the effects on distortion of the DNA double helix structure by binding of actinomycin and triostin are quite different, since actinomycin and triostin are mono- and bis-intercalators, respectively. In spite of such significant differences in the binding sites and the effects of drug induced unwinding of the helix ( $26^{\circ}$ for actinomycin ${ }^{12)}$ and $47^{\circ}$ for triostin ${ }^{13)}$ ), the biological effects of actinomycin and triostin are generally identical. The most striking biological action of actinomycin is its selectivity as an inhibitor of DNA-directed RNA synthesis ${ }^{14)}$. For example, actinomycin inhibits the activity of Escherichia coli RNA polymerase at actinomycin/DNA ratios far below those required to inhibit the activity of DNA polymerase $\mathrm{I}^{15,16)}$. Quinomycin and triostin have biological effects reminiscent of actinomycin. They are extremely powerful, selective inhibitors of DNA-directed RNA synthesis in vivo and in vitro ${ }^{17,18)}$. The inhibition mechanisms of actinomycin, quinomycin and triostin are obviously different from those of simple intercalator drugs such as proflavine, which inhibits RNA synthesis at the level of initiation of transcription ${ }^{19,20)}$. It has been believed that the antibiotics act primarily by slowing down the chain elongation reaction ${ }^{14)}$. Kinetic studies indicated that the enzyme must wait for the drug to dissociate away before it can progress further along its template ${ }^{21}$. Thermodynamic ${ }^{22)}$ and kinetic studies ${ }^{21)}$ have suggested that the cyclic depsipeptide rings are important for slowing down the rate of the dissociation of the complex.

We now ask, do the cyclic depsipeptide rings of these antibiotics bear any mysterious code which the RNA polymerase recognizes as a special signal? We propose a hypothesis for the role of the cyclic 
Fig. 5. Schematic illustration of the proposed transcription procedure at the binding site of actinomycin.

The RNA polymerase is omitted in this figure. The small solid horizontal rectangle and two large solid vertical rectangles represent the phenoxazone ring and two cyclic depsipentapeptide rings of actinomycin molecule, respectively. The characteristic carbonyl oxygen atoms on the surfaces of cyclic depsipeptide rings are indicated by $O$.

The DNA is shown by open rectangles (basepair) with solid lines (back-bone). The five basepairs from the bottom of figure are already opened by the unwinding activity of the RNA polymerase. The transcript is indicated by several squares connected by solid line.

The letters in the squares represent the RNA component from DNA template. The consecutive $U$ sequence terminates the transcription and release the premature RNA transcript from the enzyme (see text).

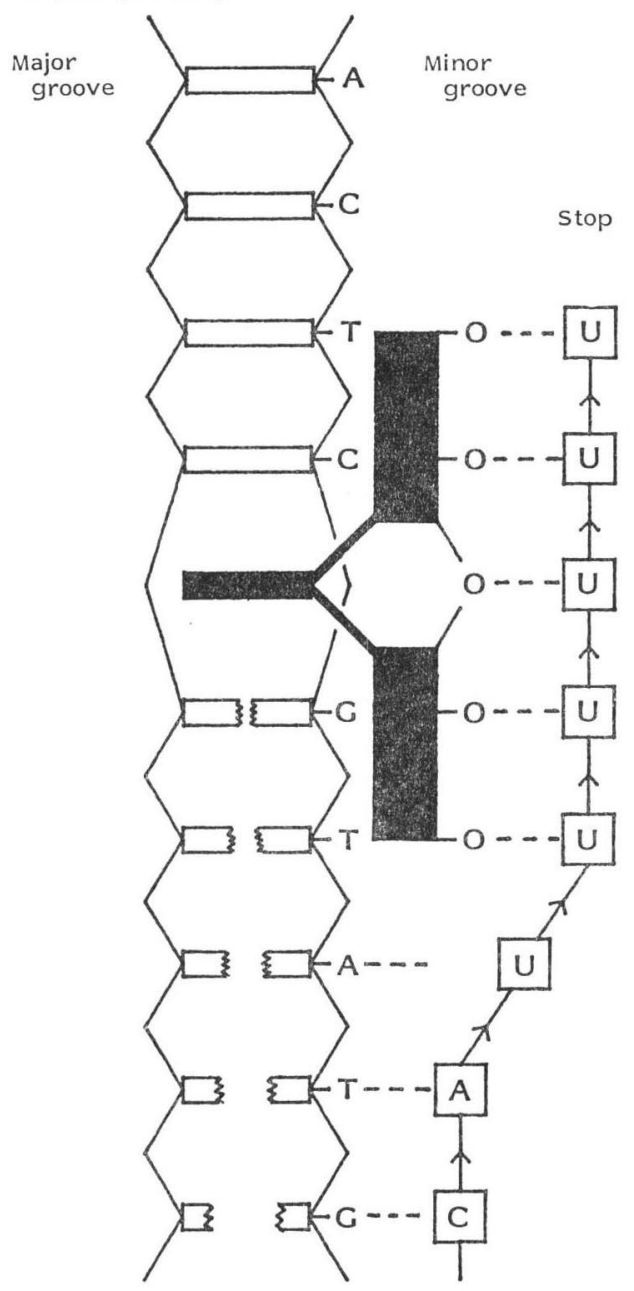

depsipeptide rings, based on the three-dimensional structures of DNA-antibiotic complexes and on recent new biological evidence ${ }^{23)}$; when the antibiotics bind in the region around the pause or rho-dependent termination sites on the DNA, the drugs terminate transcription and cause release of a premature RNA transcript, rather than merely slowing down the elongation reaction.

Both actinomycin and triostin bind in the minor groove of the DNA double helix. The binding sites of both antibiotics contain G-C base pairs which are completely covered by the cyclic depsipeptide rings of the antibiotics. The characteristic features of the outer surface of the cyclic depsipeptide rings of both antibiotics are the extremely similar. The carbonyl oxygen atoms of the amino acid residues are located on the outer surface of cyclic depsipeptide rings. Specifically, in the actinomycin D complex (Fig. 2), two $\mathrm{O}$ (proline)-O(sarcosine) $(4.2 \AA)$ pairs and one O(D-valine)-O(D-valine) $(4.5 \AA)$ pair are approximate on the adjacent base-pairs of the intercalation site and on the chromophore of the antibiotic, respectively. In addition, the carbonyl oxygen atom of $N$-methyl-L-valine residue locates near on the G-C base pair. Similarly, in the triostin A complex (Fig. 3), two $\mathrm{O}(\mathrm{D}$-serine)-O(N-methyl-L-cystine) pairs and two carbonyl oxygen atoms of $N$-methyl-L-valine residues are near on the G-C base pairs and on the chromophores of the antibiotic, respectively. Two sulfur atoms in the disulfide bridge are also on the outer surface and locate approximately center of the ring. The distances of the oxygenoxygen pairs are approximately equal to the distance, $4.7 \AA^{24)}$, between the N3 of adenine and the $\mathrm{O} 2$ of thymine of a Watson-Crick A-T base pair (Fig. 4). If the surfaces of the cyclic depsipeptides are considered in terms of hydrogen-bond donor and/or acceptors, they are therefore quite similar to the surface of the minor groove of a DNA helix. Further, with both 
actinomycin and triostin, the surface of the rings simulate a poly A-T base-pair region, since there is no hydrogen bond donor group on the surfaces. Such a donor would be present in a G-C containing region.

When the actinomycin binds to the DNA, the surface of the minor groove is covered for an approximately $14 \AA$ which corresponds to the five base-pairs being covered by cyclic depsipeptide rings whose outer surfaces carry the five consecutive, apparent A-T base-pairs (Fig. 2). For the quinoxaline antibiotics such as triostin and quinomycin, the corresponding numbers are $11 \AA$ and four base-pairs (Fig. 3). But, quinoxaline antibiotics bind preferentially at the ACGT and TCGT sequences ${ }^{11)}$, where the middle of CG sequence is sandwiched by their two chromophores and thus the both adjacent base-pair of binding site are A-T pairs. Therefore, the area of binding site of quinoxaline antibiotics has at least six consecutive A-T base-pairs in character if the outer surface of the cyclic depsipeptide ring is taken account of the four A-T base pairs.

Does this characteristic feature of the cyclic depsipeptide rings have any biological meaning? If the RNA polymerase reads the DNA template along the minor groove of DNA before the unwinding activity breaks the base-pair hydrogen bonds, then it must recognize a $\mathrm{G}-\mathrm{C}$ base pair by the sequence hydrogen bond acceptor $(\mathrm{N}:$ ), donor $(\mathrm{NH})$ and acceptor (O:), and an A-T base-pair by acceptor $(\mathrm{N}:)$ and acceptor $(\mathrm{O}:)$ as shown in Fig. $4^{25)}$. Thus, the surfaces of the cyclic depsipeptide rings of actinomycin, quinomycin and triostin, as seen by RNA polymerase would be recognized from their acceptor-acceptor sequence as a poly A-T base-pairs region.

For rho-independent termination of RNA polymerase, the causative factor appears to be a hyphenated dyad symmetry element followed by five to seven $\mathrm{A} / \mathrm{T}$ residues ${ }^{26} 28$. Most pause sites are also found to contain elements of symmetry centered $16 \sim 19$ nucleotides upstream from the pause ${ }^{29)}$. The pause sites do not release the RNA transcript from the enzyme when the characteristic series of A/T's after the symmetry elements is lacking in the template DNA. But when an antibiotic such as actinomycin or quinomycin or triostin binds near such a pause site, the site could become a rho-independent termination site, because of the deceptive sequence of five to six consecutive, apparent A-T base pairs carried on the surface of the cyclic depsipeptide rings (Fig. 5).

Alternatively, for rho-dependent termination, the termination sites are quite similar to the pause sites; both contain the capacity for hairpin formation ${ }^{30 ~ 322}$. Although the mechanism of rho action is unclear, it could be that a antibiotic such as actinomycin or quinomycin or triostin binds near the rho-dependent termination site or the pause site, and it behaves like a rho itself. In summary, actinomycin and quinoxaline antibiotics appear to change the nature of normal pause or rho-dependent termination sites to an abnormal termination site by covering the site of minor groove of DNA with cyclic depsipeptides specially designed by nature.

This hypothesis rests on many speculations. However, it is consistent with biological data which cannot be explained by the current hypothesis that the antibiotics simply slow down the chain elongation reaction. An example is a recent elegant experiment performed by GAINES and ATTARDi ${ }^{23)}$. Using isolated HeLa cell mitochondria, they found that mitochondrial RNA synthesis was fairly sensitive to actinomycin D. Particularly noticeable was the effect of the drug on the forming of 16S RNA, which was almost totally abolished at a concentration of actinomycin $\mathrm{D}$ of $0.8 \mu \mathrm{M}$. The decline in the 16S RNA was accompanied by an increase in a relative component which was identified by RNA sequencing to correspond to the proximal one-third of 16S RNA. Densitometric tracings indicated that this new component could account for a major portion of the decrease of the 16S RNA. In 
addition, several small-sized RNA's were also observed to increase significantly. These could be premature fragments of large-sized RNA's such as 12S RNA. Premature fragments were not observed in inhibition by either simple intercalating drugs such as proflavin, ethidium bromide and ellipticine or in inhibition by lowering of the temperature.

A second example is the biological inactivity of the synthetic analogue, de- $N$-tetramethyltriostin $\mathrm{A}^{33)}$. This substance binds to the DNA as strongly as triostin A itself, and even more strongly to AT-rich $\mathrm{DNA}^{7)}$. The unwinding angle $\left(46^{\circ}\right)$ of the DNA helix due to intercalation of this analogue indicates that the analogue is a bis-intercalator, just as triostin $\mathrm{A}$ is (unwinding angle $\left.47^{\circ}\right)^{7,13)}$. However, no significant biological activity has been reported about this synthetic analogue ${ }^{7,33)}$. If the conformation of the cyclic depsipeptide in the DNA complex is similar to that of triostin $\mathrm{A}^{3)}$, the amide hydrogen $(\mathrm{NH})$ of the L-cystine residue should be on the surface of the cyclic depsipeptide ring. The amide hydrogens point approximately towards the outer surface in the crystal structure of de- $N$ tetramethyltriostin $\mathrm{A}$ itself ${ }^{34)}$. The presence of this amide hydrogen on the surface could change the nature of hydrogen bonding scheme, providing a hydrogen-bond donor as with G-C and thus destroying the perfect A-T character seen with the natural antibiotics. The cyclic depsipeptide of this analogue would lose the special coding for termination of the RNA polymerase action, which we asummed for triostin $\mathrm{A}$.

\section{Acknowledgment}

We wish to express our thanks to Professors R. L. Schowen, K. B. Mertes, A. W. Burgstahler and G. MAGGIORA of our Department for a critical reading of the manuscript and very valuable comments. Thanks are also due to Dr. H. M. Berman for valuable discussion.

\section{References}

1) Takusagawa, F. \& H. M. Berman: Some aspects of actinomycin D-nucleic acid binding. Cold Spring Harbor Symp. Quant. Biol. 37: 315 321, 1983

2) Takusagawa, F.; M. Dabrow, S. Neidle \& H. M. Berman: The structure of a pseudo intercalated complex between actinomycin and the DNA binding sequence $d(\mathrm{GpC})$. Nature 296: 466 469, 1982

3) Wang, A. H.-J.; G. Ughetto, G. J. Quigley, T. Hakoshima, G. A. van DeR Marel, J. H. van Boom \& A. Rich: The molecular structure of a DNA-triostin A complex. Science 225: 1115 1121, 1984

4) Sobell, H. M. \& S. C. JAIN: Stereochemistry of actinomycin binding to DNA. II. Detailed molecular model of actinomycin-DNA complex and its implications. J. Mol. Biol. 68:21 34, 1972

5) Sobell, H. M.; S. C. Jain, T. D. SAKore \& C. E. Nordman: Stereochemistry of actinomycin-DNA binding. Nature New Biol. 231: 200 205, 1971

6) Kyogoku, Y.; N. Higuchi, M. Watanabe \& K. Kawano: Conformer equilibria of triostin A and its conformer-specific interaction with nucleic acid bases. Biopolymers 20: 1959 1970, 1981

7) LeE, J.S. \& M. J. WARING: Interaction between synthetic analogues of quinoxaline antibiotics and nucleic acids. Biochem. J. 173: 129 144, 1978

8) Fox, K. R.; R. K. OLSEN \& M. J. WARING: Interaction between synthetic analogues of quinoxaline antibiotics and nucleic acids: Role of the disulphide cross-bridge and D-amino acid centres in des- $N$ tetramethyl-triostin A. Brit. J. Pharmacol. 70: 25 40, 1980

9) Meienhofer, J. \& E. Atherton: Structure-activity relationships in the actinomycins. In Structureactivity Relationships among the Semisynthetic Antibiotics. Ed., D. Perlman, pp. 427 529, Academic Press, New York, 1977

10) Scamrov, A. V. \& R. Sh. Beabfalashvilli: Binding of actinomycin D to DNA revealed by DNase I footprinting. FEBS Lett. 164: 97 101, 1983

11) Van Dyke, M. M. \& P. B. Dervan： Echinomycin binding sites on DNA. Science 225: 1122 1127, 1984

12) WARING, M.: Variation of the supercoils in closed circular DNA by binding of antibiotics and drugs: Evidence for molecular models involving intercalation. J. Mol. Biol. 54: 247 279, 1970 
13) LeE, J. S. \& M. J. WARING: Bifunctional intercalation and sequence specificity in the binding of quinomycin and triostin antibiotics to deoxyribonucleic acid. Biochem. J. 173: 115 128, 1978

14) Gale, E. F.; E. Cundliffe, P. E. Reynolds, M. H. Richmond \& M. J. Waring: The Molecular Basis of Antibiotic Action. Wiley \& Sons, New York, 1981

15) Hurwitz, J.; J. J. Furth, M. Malamy \& M. Alexander: The role of deoxyribonucleic acid in ribonucleic acid synthesis. III. The inhibition of the enzymatic synthesis of ribonucleic acid and deoxyribonucleic acid by actinomycin D and proflavin. Proc. Natl. Acad. Sci. U.S.A. 48: 1222 1223, 1962

16) Reich, E. \& I. H. Goldberg: Actinomycin and nucleic acid function. Progress in Nucleic Acid Res. \& Mol. Biol. 3: 183 234, 1964

17) Katagiri, K.; T. Yoshida \& K. Sato: Quinoxaline antibiotics. In Antibiotics. III. Mechanism of Action of Antimicrobial and Antitumor Agents. Ed., J. W. CorCORAN et al., pp. 234 251, 1975

18) Waring, M. \& A. Makoff: Breakdown of pulse-labeled ribonucleic acid and polysomes in Bacillus megaterium: Actions of streptolydigin, echinomycin and triostins. Mol. Pharmacol. 10: 214 224, 1974

19) Richardson, J. P.: The binding of RNA polymerase to DNA. J. Mol. Biol. 21: 83 114, 1966

20) Richardson, J. P.: Mechanism of ethidium bromide inhibition of RNA polymerase. J. Mol. Biol. 78: $703 \sim 714,1973$

21) Muller, W. \& D. M. Crothers: Studies of the binding of actinomycin and related compounds to DNA. J. Mol. Biol. 35: $251 \sim 290,1968$

22) Gellert, M.; C. E. Smith, D. Neville \& G. Felsenfeld: Actinomycin binding to DNA: Mechanism and specificity. J. Mol. Biol. 11: 445 457, 1965

23) Gaines, G. \& G. AttARdi: Intercalating drugs and low temperatures inhibit synthesis and processing of ribosomal RNA in isolated human mitochondria. J. Mol. Biol. 172: 451 466, 1984

24) Viswamitra, M. A.; Z. Shakked, P. G. Jones, G. M. Sheldrick, S. A. Salisbury \& O. Kennard: Structure of the deoxytetranucleotide d-pApTpApT and a sequence-dependent model for poly(dA-dT). Biopolymers 21: $513 \sim 533,1982$

25) Seeman, N. C.; J. M. Rosenberg \& A. Rich: Sequence-specific recognition of double helical nucleic acids by proteins. Proc. Natl. Acad. Sci. U.S.A. 73: 804 808, 1976

26) Rosenberg, M. \& D. Court: Regulatory sequences involved in the promotion and termination of RNA transcription. Annu. Rev. Genet. 13: 319 353, 1979

27) Farnham, P. J. \& T. Platt: A model for transcription termination suggested by studies on the trp attenuator in vitro using base analogs. Cell 20: 739 748, 1980

28) Farnham, P. J. \& T. Platt: Rho-independent termination: Dyad symmetry in DNA causes RNA polymerase to pause during transcription in vitro. Nucleic Acids Res. 9: 563 577, 1981

29) Gamper, H. B. \& J. E. Hearst: A topological model for transcription based on unwinding angle analysis of $E$. coli RNA polymerase binary, initiation and ternary complexes. Cell 29: 81 90, 1980

30) Küpper, H.; T. Sekiya, M. Rosenberg, J. Egan \& A. Landy: A $\rho$-dependent termination site in the gene coding for tyrosine tRNA su ${ }_{3}$ of Escherichia coli. Nature 272: 423 428, 1978

31) Rosenberg, M.; D. Court, H. Shimatake, C. Brady \& D. L. Wulff: The relationship between function and DNA sequence in an intercistronic regulatory region in phage $\lambda$. Nature 272: 414 423, 1978

32) Calva, E. \& R. R. Burgess: Characterization of a $\rho$-dependent termination site within the cro gene of bacteriophage $\lambda$. J. Biol. Chem. 255: 11017 11022, 1980

33) Ciardelli, T. L. \& R. K. Olsen: Synthesis of des- $N$-tetramethyltriostin A, a bicyclic octadepsipeptide related to the quinoxaline antibiotics. J. Am. Chem. Soc. 99: 2806 2807, 1977

34) Hossain, M. B.; D. van der Helm, R. K. Olsen, P. G. Jones, G. M. Sheldrick, E. Egert, O. Kennard, M. J. WARING \& M. A. Viswamitra: Crystal and molecular structure of the quinoxaline antibiotic analogue TANDEM (des- $N$-tetramethyltriostin A). J. Am. Chem. Soc. 104: $3401 \sim 3408,1982$ 